\title{
Stereo Vision-based Autonomous Navigation for Oil and Gas Pressure Vessel Inspection Using a Low-cost UAV*
}

\author{
Leijian $\mathrm{Yu}^{1}$, Erfu Yang ${ }^{1,2}$, Beiya Yang $^{1}$, Andrew Loeliger ${ }^{1}$ and Zixiang Fei $^{1}$
}

\begin{abstract}
It is vital to visually inspect pressure vessels regularly in the oil and gas company to maintain their integrity. Compared with visual inspection conducted by sending engineers and ground vehicles into the pressure vessel, utilising an autonomous Unmanned Aerial Vehicle (UAV) can overcome many limitations including high labour intensity, low efficiency and high risk to human health. This work focuses on enhancing some existing technologies to support low-cost UAV autonomous navigation for visual inspection of oil and gas pressure vessels. The UAV can gain the ability to follow the planned trajectory autonomously to record videos with a stereo camera in the pressure vessel, which is a GPS-denied and low-illumination environment. Particularly, the ORB-SLAM3 is improved by adopting the image contrast enhancement technique to locate the UAV in this challenging scenario. What is more, a vision hybrid Proportional-Proportional-Integral-Derivative (P-PID) position tracking controller is integrated to control the movement of the UAV. The ROS-Gazebo-PX4 simulator is customised deeply to validate the developed stereo vision-based autonomous navigation approach. It is verified that compared with the ORBSLAM3, the numbers of ORB feature points and effective matching points obtained by the improved ORB-SLAM3 are increased by more than $400 \%$ and $600 \%$, respectively. Thereby, the improved ORB-SLAM3 is effective and robust enough for UAV self-localisation, and the developed stereo vision-based autonomous navigation approach can be deployed for pressure vessel visual inspection.
\end{abstract}

\section{INTRODUCTION}

Pressure vessels are major assets in oil and gas companies and have a wide range of applications such as being storage containers, separators and filters. Their failures can result in catastrophe to both the natural environment and human safety. Thus, regular visual inspection of these infrastructures is required [1]. Visual inspection of oil and gas pressure vessels has been traditionally performed by engineers deployed to pressure vessels or operating a telepresent ground vehicle [2]. The former method puts engineers' life and health at risk, while the latter is time-consuming due to its low mobility and operation complexities. Instead, using an autonomous Unmanned Aerial Vehicle (UAV) can achieve a close view of the surface inside the pressure vessel with a high degree of manoeuvrability [3].

Some reviews about UAV autonomous navigation techniques for inspection tasks can be seen in [4]. Among them, reliable

\footnotetext{
*This work is supported in part by the UK Oil and Gas Technology Centre (OGTC) under the LOCUST research project (2019-2021, Grant No.: AI-P-028). Mr. Leijian Yu is funded by the China Scholar Council and the International Fees Only Studentship from the University of Strathclyde (2018-2021)

${ }^{1}$ Leijian Yu, Erfu Yang, Beiya Yang, Andrew Loeliger and Zixiang Fei are with the Department of Design, Manufacturing and Engineering Management, University of Strathclyde, Glasgow, G1 1XJ, United Kingdom.

${ }^{2}$ Corresponding author. E-mail: erfu.yang@strath.ac.uk
}

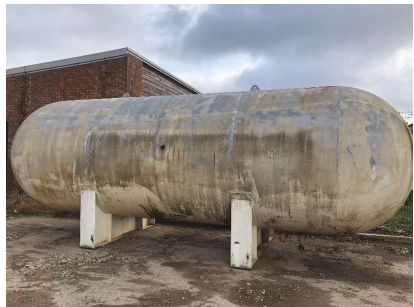

(a)

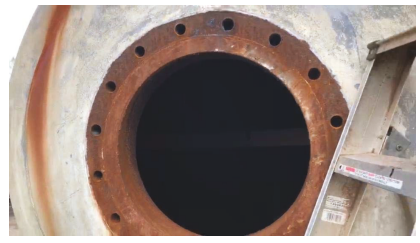

(b)

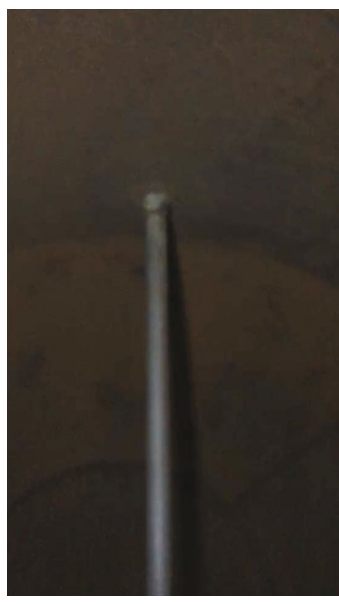

(c)
Fig. 1. One pressure vessel. (a) The overview of the pressure vessel; (b) The entrance of the pressure vessel; (3) The inside of the pressure vessel.

state or vehicle pose estimation without GPS signal is one of the most important research areas. In UAV-based indoor visual inspection applications, the Light Detection and Ranging (LiDAR) sensor is the most popular one. In [5], Castaño et al. demonstrated a semi-autonomous UAV system for sewerage inspection. The UAV utilised four TeraRanger One sensors to compute its position relative to the pipe longitudinal axis, and the UAV was remotely controlled by engineers. The autonomous UAV navigation system developed by [6] equipped with the RPLiDAR A3 to perform 2D localisation for autonomous monitoring tunnel infrastructures. Özaslan et al. proposed a UAV navigation system that utilised a Velodyne Puck 3D LiDAR to estimate its position and conducted inspection tasks for Penstocks and Tunnels [7]. Nevertheless, the low-cost LiDAR cannot support precise UAV autonomous 3D navigation, while a precise 3D LiDAR runs against the low-cost feature of the platform [8].

In the robotic navigation and infrastructure inspection literature, the visual Simultaneous Localisation and Mapping (SLAM) has shown great prospect, since it can preciously perform the estimation of the state of a robot [9]. Monocular visual-inertial SLAM enabled autonomous navigation approaches for aerial visual inspection of the Hardturm Stadium and transmission towers were demonstrated by Teixeira et al. [10] and Bian et al. [11], respectively. To improve the necessary robustness of the navigation system, Nikolic et al. proposed an off-board stereo visual-inertial SLAM method 
to estimate the pose of the UAV and achieved the navigation capability inside the industrial boiler to perform inspection tasks [12]. However, the cable limits the movement of the UAV. What is more, these autonomous navigation systems rely on the integrated visual and time-synchronised inertial sensors to realise the UAV ego-motion estimation. The highquality inertial measurement unit (IMU) adds extra cost and payload to the low-cost UAV platform.

This study deals with the pressure vessel which is a GPSdenied and low-illumination environment (as shown in Fig. 1). Autonomous navigation for low-cost UAV-refined inspection inside the pressure vessel is really challenging and has not been fully implemented. Thus, this work proposes a novel stereo vision-based autonomous navigation approach for the automatic acquisition of images inside oil and gas pressure vessels with a low-cost UAV. The main contributions are summarised as follows.

- The ORB-SLAM3 is improved by the image contrast enhancement capability to estimate the pose of the UAV in the GPS-denied and low lighting environment.

- The stereo vision-based autonomous navigation approach is developed which allows the low-cost UAV to track the planned inspection trajectory stably inside the pressure vessel.

- The ROS-Gazebo-PX4 simulator is customised deeply to support UAV autonomous navigation performance evaluation in the oil and gas pressure vessel visual inspection scenario. The effectiveness of the proposed approach is demonstrated in the developed simulation environment.

The rest of the paper is organised as follows. Details of the proposed stereo vision-based autonomous navigation approach are given in Section II. In Section III, the experimental environment is demonstrated. Section IV shows and analyses the performance of the proposed approach. Finally, conclusions and future work are provided in Section V.

\section{ApPROACH SCHEME DESCRIPTION}

The scheme of the proposed stereo vision-based autonomous navigation approach is shown in Fig.2. Based on the images captured by the UAV on-board stereo camera, the UAV will locate itself through the visual localisation technology. Then, compared with the planned trajectory, the next target position will be updated. Afterwards, the proper control signal will be generated to control the UAV to move to the target position. The whole process will be repeated until the UAV finishes the whole trajectory.

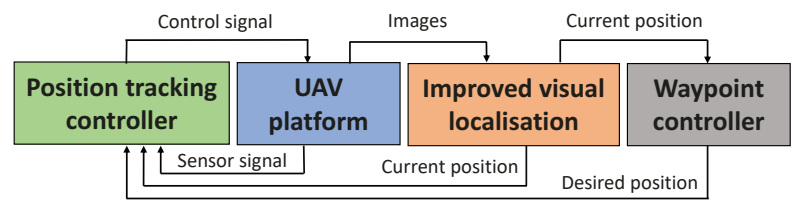

Fig. 2. Scheme of the vision-based autonomous navigation approach.

\section{A. Improved Visual Localisation}

To navigate successfully in a GPS-denied and lowillumination environment, the UAV must have the selflocalisation capability. The ORB-SLAM3 [13] is claimed as the robustness visual SLAM system in the literature, and it has three main threads, i.e. tracking, local mapping, and loop and map merging. Besides, it utilises the multi-map technology named Atlas to improve the robustness of the system. However, the ORB-SLAM3 relies on matching ORB feature point pairs heavily. In the low lighting environment, the number of stable ORB feature points drops significantly. Thus, the system fails to obtain enough input information, and posture cannot be calculated and corrected. When ORB feature points are less than 500 in all frames, the pose estimation process cannot be performed so that the initialisation and tracking fail [14].

Image pre-processing technologies have been used widely to improve the performance of computer vision tasks, such as the image sharpening technique for facial emotion recognition [15] and speckle reduction for synthetic aperture radar (SAR) image recognition [16]. In the oil and gas pressure vessel inspection scenario, the light is insufficient to the ORB-SLAM3, and the image contrast enhancement technology improves the visual quality of dimmed images. Thus, the image contrast enhancement method is adopted before the ORB feature point extraction process in the tracking thread. The image contrast enhancement technology makes the image contain more prominent textures, thereby increasing the number of stable ORB feature points. Eventually, the robustness and stability of the ORB-SLAM3 are improved.

1) Atlas: Atlas is a multi-map representation that contains all non-active maps in the ORB-SLAM3. The active map is utilised by the tracking thread to locate the incoming frames. All other maps are treated as disconnected maps and stored in Atlas. When the system re-enters the mapped scene, the active map will be merged with the relative non-active map.

2) Tracking Thread: The tracking thread processes each frame of the video. Firstly, it will initialise the system with the first frame that can be extracted more than 500 ORB feature points. Then, the system starts tracking the pose of the current frame. When the tracking fails, the relocation function is activated to relocate the current frame in all the maps. If being relocated, the corresponding map becomes the active map. Otherwise, a new active map is created while all other maps are stored in Atlas as non-active maps. The bundle adjustment is applied to process the active map points to minimise the reprojection error and optimise the pose of the current frame. If the current frame meets certain conditions, it will be selected as a keyframe.

3) Local Mapping Thread: Keyframes generated by the tracking thread are sent to the local mapping thread. The newly added keyframe and corresponding map points are inserted into the active map. A local bundle adjustment is performed to optimise the poses of map points and keyframes. To maintain the size of the map, the redundant map points and keyframes will be deleted. 
4) Loop and Map Merging Thread: The input of this thread is the refined keyframes by the local mapping thread. It detects the overlap scenes between the active map and Atlas. If it exists in different maps, the active map and matching map will be merged as a new active map. Otherwise, the loop closure is utilised in the active map. When the loop correction is finished, an independent thread executes a global bundle adjustment to reduce the accumulated drift error.
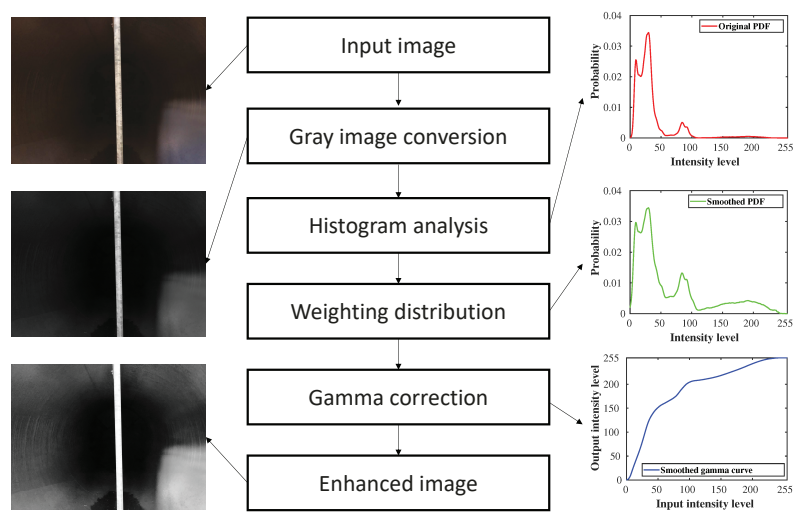

Fig. 3. Diagram of the adaptive gamma correction with weighting distribution.

5) Adaptive Image Enhancement: In the image contrast enhancement domain, considering the easy adjustment and efficient implementation capabilities, gamma correction [17] has been widely utilised. It enhances image contrast by directly modifying pixel values based on regulation. An adaptive gamma correction algorithm with weighting distribution [18] is adopted to process frames before the ORB feature points extraction procedure. Fig. 3 shows the steps for enhancing the image in high level. As ORB feature points are extracted from the gray image, the colour image needs to be transformed to the gray image first. In the dimmed gray image, most of pixels lie in the low-intensity level. After the weighting distribution and gamma correction, more pixels will be distributed in the high-intensity region, thereby improving image contrast.

Specifically, gamma correction techniques use the parameter $\gamma$ to adjust the luminance of the image. The transform-based gamma correction is represented by

$$
F(l)=l_{\max }\left(\frac{l}{l_{\max }}\right)^{\gamma}
$$

where $l$ and $l_{\max }$ represent the intensity of each pixel and the maximum intensity in the input image, respectively. In (1), the highest intensity in the output image is restricted by the maximum intensity of the input image. To deploy it in a low-illumination environment, the $l_{\max }$ is set to 255 , which is the highest intensity value in the gray image. Thus, the modified gamma correction can be defined as

$$
F(l)=255\left(\frac{l}{255}\right)^{\gamma}
$$

The Probability Density Function (PDF) can be approximated by

$$
P D F(l)=\frac{n_{l}}{N}
$$

where $n_{l}$ represents the number of pixels that have intensity $l . N$ denotes the total number of pixels in the image. Based on the PDF, the Cumulative Distribution Function (CDF) can be formulated as

$$
C D F(l)=\sum_{k=0}^{l} P D F(k)
$$

The adaptive gamma correction is written as

$$
F(l)=255\left(\frac{l}{255}\right)^{(1-C D F(l))}
$$

Additionally, the weighting distribution function is utilised to modify the statistical histogram with less adverse effects. The weighting distribution function is expressed as

$$
P D F_{w d}(l)=P D F_{\max }\left(\frac{P D F(l)-P D F_{\min }}{P D F_{\max }-P D F_{\min }}\right)^{\alpha}
$$

where $\alpha$ indicates the adjusted parameter, and it is 0.5 in this work. $P D F_{\max }$ and $P D F_{\min }$ denote the maximum and minimum $P D F$ of the statistical histogram, respectively. So, the modified CDF is defined as

$$
C D F_{w d}(l)=\frac{\sum_{l=0}^{l_{\max }} P D F_{w d}(l)}{\sum P D F_{w d}}
$$

where the sum of $P D F_{w d}$ is represented as follows

$$
\sum P D F_{w d}=\sum_{l=0}^{l_{\max }} P D F_{w d}(l)
$$

Finally, the $\gamma$ parameter can be calculated by

$$
\gamma=1-C D F_{w d}(l)
$$

To reduce the computational complexity, the temporal technique is applied. The information content contained by each frame is represented by the following entropy function

$$
H=-\sum_{l=0}^{l_{\max }} P D F(l) \log (P D F(l))
$$

The differences between the information contents contained by two frames can be defined as

$$
T_{h}=\left|H_{\text {current }}-H_{\text {previous }}\right|
$$

The first frame is stored and utilised to calculate the $\gamma$ transformation curve. When the $T_{h}$ exceeds 0.05 , the stored frame is updated with the current frame. At the same time, the $\gamma$ transformation curve is modified. Otherwise, the existing $\gamma$ transformation curve is applied directly to transform the intensity level of the incoming frame. 


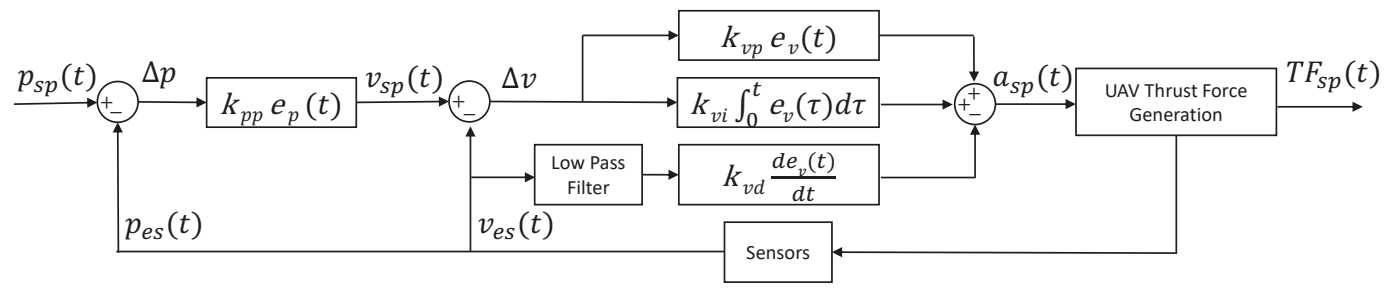

Fig. 4. Scheme for position tracking controller.

\section{B. Position Tracking Controller}

After the UAV is located by the improved ORB-SLAM3, the waypoint controller compares the current position with the planned inspection path to compute the desired position. Afterwards, a position tracking controller is needed to control the movement of the UAV.

In this section, a vision hybrid position tracking controller is developed from the PX4 [19], the leading open-source autopilot stack for the UAV, to realise an accurate position control mechanism for the UAV. The position tracking controller is demonstrated in Fig. 4. Herein, based on the PID control law, a $\mathrm{P}$ loop for position error $\left(e_{p}(t)\right)$ and a PID loop for velocity error $\left(e_{v}(t)\right)$ are cascaded as the position tracking controller. The PID velocity control loop contains three parameters, taken as constant $k_{v p}, k_{v i}$ and $k_{v d}$ which are responsible to adjust the proportional, integral and differential unit, respectively. Its continuous form can be given as

$$
a_{s p}(t)=k_{v p} e_{v}(t)+k_{v i} \int_{0}^{t} e_{v}(\tau) d \tau+k_{v d} \frac{d e_{v}(t)}{d t} e_{v}(t)
$$

The UAV is controlled by a digital controller operating in a sampled-data feedback loop. Define

$$
\begin{aligned}
& z_{i}(k)=z_{i}(k-1)+z(k) \\
& z_{d}(k)=z(k)-z(k-1)
\end{aligned}
$$

where $z(k)$ represents an error variable. $z_{i}(k)$ is the integrator state, and $z_{d}(k)$ indicates the differentiator state. Define the discrete-time PID controller as

$$
a_{s p}(k)=k_{v p}(k) z(k)+k_{v i}(k) z_{i}(k)+k_{v d}(k) z_{d}(k)
$$

Equation (15) can be rewritten as

$$
a_{s p}(k)=\theta_{v}(k) \phi_{v}(k)
$$

where

$$
\begin{gathered}
\theta_{v}(k) \triangleq\left[k_{v p}(k) k_{v i}(k) k_{v d}(k)\right] \\
\phi_{v}(k) \triangleq\left[\begin{array}{c}
z(k) \\
z_{i}(k) \\
z_{d}(k)
\end{array}\right]
\end{gathered}
$$

By modifying $\theta$ and $\phi$, the $\mathrm{P}$ position control can be represented as

$$
v_{s p}(k)=k_{p p}(k) z_{p}(k)
$$

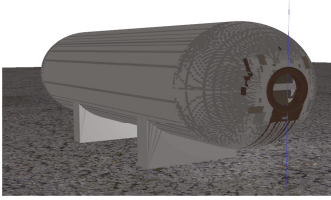

(a)

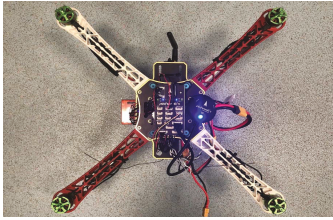

(c)

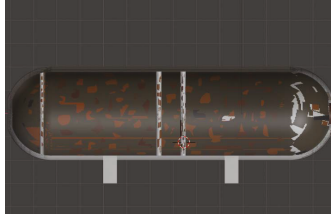

(b)

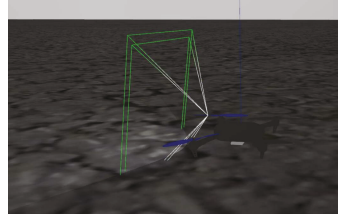

(d)

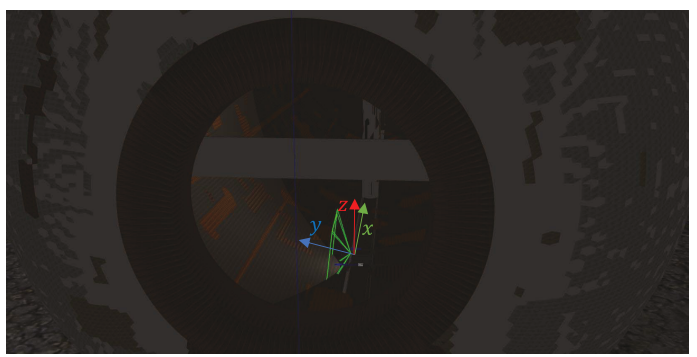

(e)

Fig. 5. Physical and simulation components. (a) The simulated pressure vessel; (b) The section view of the simulated pressure vessel; (c) The physical UAV; (d) The simulated UAV equipped with a stereo camera and a spotlight source; (e) The developed simulation environment.

\section{EXPERIMENTAL ENVIRONMENT}

Due to the high risk of property damage and battery constraints, extensive physical UAV flying tests are costly and time-consuming. As an alternative solution, simulation allows to test and validate the developed algorithm in "realistic" scenarios, which can avoid the potential risk in real flights. In the UAV research domain, the Robot Operating System (ROS) [20] is the most popular and convenient middleware suite. Moreover, it comes with the Gazebo simulator [21] that contains a physics engine to imitate actual motions of the UAV in the customised environment. Thus, the simulation environment developed in this paper is based on the ROS-Gazebo-PX4 toolchain. In general, Gazebo contains a simulated pressure vessel and a simulated UAV model. The UAV model is based on the PX4 firmware for the dynamic simulation. The PX4 communicates with the Gazebo to receive sensor data from the simulated world and send the motor commands back. Meanwhile, all the 
components are coordinated through ROS.

Specifically, based on the pressure vessel shown in Fig. 1, a pressure vessel model is constructed (as shown in Fig. $5(\mathrm{a})$ ). Its dimension is $7 \mathrm{~m} \times 2.5 \mathrm{~m} \times 2.5 \mathrm{~m}$. As demonstrated in Fig. 5(b), it contains shells, several horizontal bars and vertical pipes. To simulate the customised quadrotor shown in Fig. 5(c), a simulated UAV is developed from the PX4 flight control stack, and it is equipped with a stereo camera and a spotlight source. Due to the horizontal bar at the entrance of the pressure vessel, the UAV is placed inside the pressure vessel. The overall layout of the simulation environment is illustrated in Fig.5(e). Within this scenario, a world coordinate system is established. The $\mathrm{x}$-axis is supposed as the depth direction of the pressure vessel, the $y$ axis is parallel to the width direction, while the $\mathrm{z}$-axis denotes the altitude.

\section{RESUlTS}

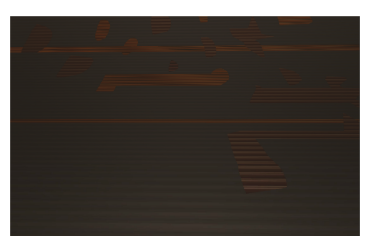

(a1)

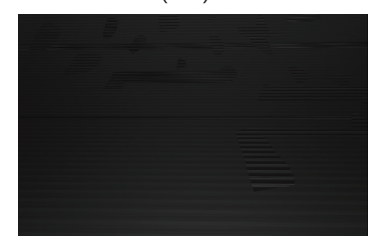

(b1)

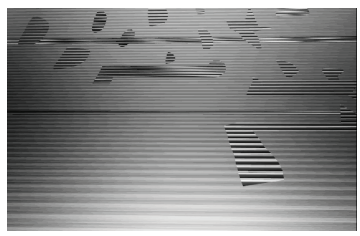

(c1)

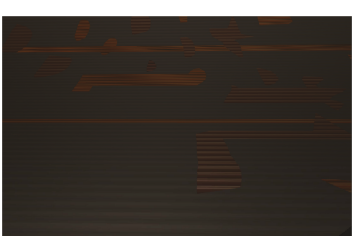

(a2)

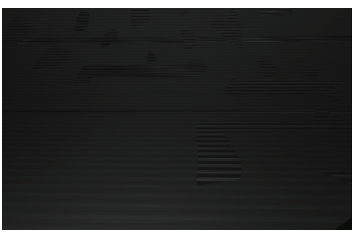

(b2)

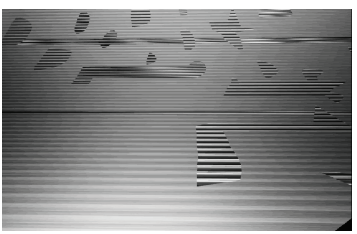

(c2)
Fig. 6. Image contrast enhancement. (a1) and (a2) are a pair of images captured by the on-board stereo camera; (b1) and (b2) are the original gray images transformed from (a1) and (a2); (c1) and (c2) are the enhanced gray images.

\section{A. Comparison of Feature Point Extraction and Matching.}

TABLE I

Comparison of Feature Point Extraction and Matching in the FIRST FRAME

\begin{tabular}{|c|c|c|c|c|}
\hline \multirow{2}{*}{ Method } & \multicolumn{2}{|c|}{ ORB feature points } & \multirow{2}{*}{$\begin{array}{l}\text { Good } \\
\text { matches }\end{array}$} & \multirow{2}{*}{$\begin{array}{c}\text { System } \\
\text { initialisation }\end{array}$} \\
\hline & Left image & Right image & & \\
\hline ORB-SLAM3 & 200 & 208 & 103 & Fail \\
\hline $\begin{array}{c}\text { Improved } \\
\text { ORB-SLAM3 }\end{array}$ & 1051 & 1092 & 746 & Success \\
\hline
\end{tabular}

In this experiment, the light intensity of the spotlight keeps the same during the whole inspection procedure and the first
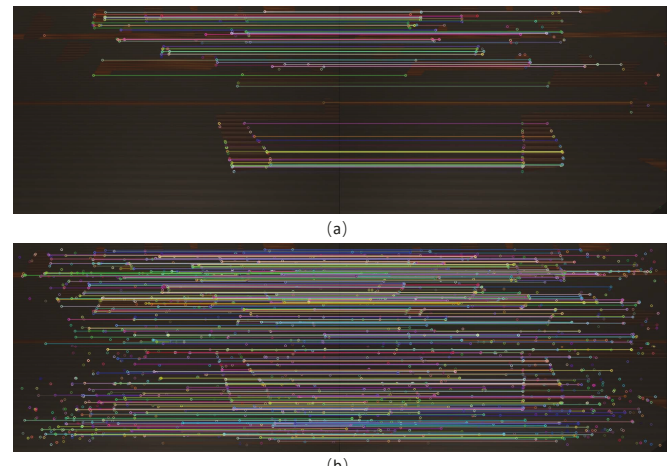

Fig. 7. Feature points extraction and matching. (a) Feature points extraction and matching based on original images; (b) Feature points extraction and matching based on enhanced images.

frame captured by the stereo camera within the developed simulation environment is selected. The results of image contrast enhancement is illustrated in Fig. 6. It is obvious that the contrast of original images is low. Within the enhanced images, the results are rich in texture information.

The ORB feature points detection and matching processes are compared using the ORB-SLAM3 with the improved ORB-SLAM3. Matches after selection through the Sum of Absolute Difference (SAD) are supposed as good matches. The visual results are shown in Fig. 7, while the statistical results are demonstrated in Table I. The ORB-SLAM3 fails to extract $500 \mathrm{ORB}$ feature points from the first frame to initialise the system. Compared to the ORB-SLAM3, more than 5 times ORB feature points can be extracted by the improved ORB-SLAM3. Meanwhile, the number of good matching points realised by the improved ORB-SLAM3 increases by $600 \%$.

The results illustrate that with the image contrast enhancement method, enough ORB feature points can be extracted to initialise the system. What is more, more effective matching points for the subsequent process as tracking, mapping and loop detection are achieved to improve the stability and robustness of the ORB-SLAM3.

\section{B. Trajectory Tracking Performance Evaluation}

The task of the aerial vehicle is to follow a pre-defined $3 \mathrm{D}$ trajectory to record videos of the pressure vessel. Fig. 8 demonstrates the 3D trajectory of the UAV and the predefined inspection plan. The square trajectory is designed to inspect the shells of the pressure vessel. What is more, the pipelines are inspected by tracking the helical path. The results indicate that keeping a constant distance to the shell in direction $y$ and $z$ is not an issue for the whole system. The parameters of the position tracking controller are adjusted for translation and shared with rotation, it negatively affects the tracking accuracy, especially in direction $x$ due to the UAV heading the $y$-axis positive direction at the beginning. The overall results validate that the UAV can locate and navigate itself stably in a pressure vessel for the visual inspection application. A supplementary video can be found at https : / / youtu.be/plzKOHhxKfI 


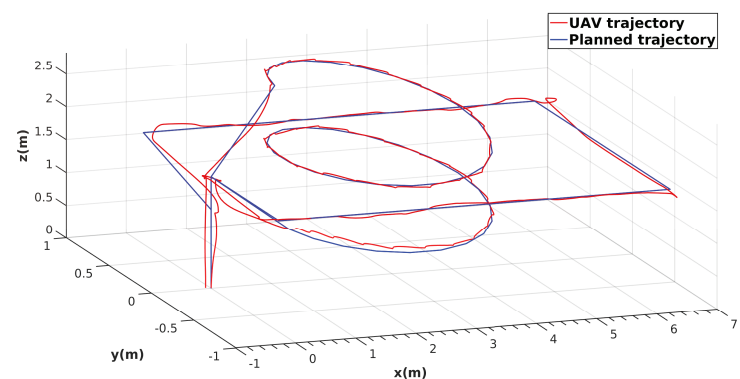

(a)

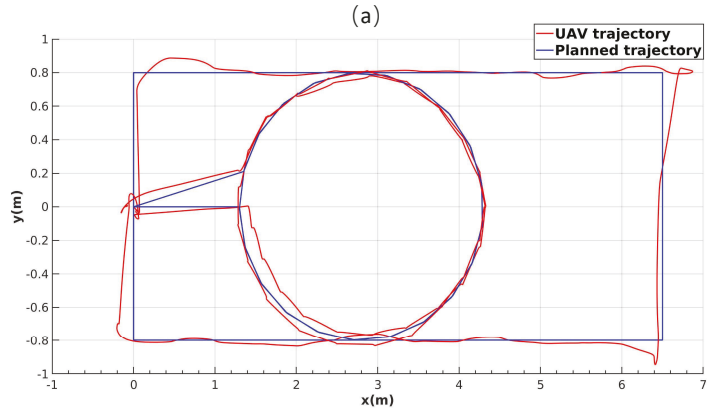

(b)

Fig. 8. Trajectory following results. (a) The overview of the UAV 3D trajectory; (b) The top view of the UAV trajectory.

\section{CONClusions And Future Work}

In this paper, a novel stereo vision-based autonomous navigation approach for low-cost aerial inspection of oil and gas pressure vessels has been presented. The key components are robust localisation in a low-illumination environment and a vision hybrid position tracking controller. The ORBSLAM3 was improved by the image contrast enhancement technique using adaptive gamma correction with weighting distribution. Besides, a P-PID controller was deployed for position tracking. The approach was successfully verified in a deeply customised ROS-PX4-Gazebo simulation environment. The results showed that the improved ORB-SLAM3 can achieve more ORB feature points and matching points than the ORB-SLAM3 in the low lighting environment, which means the effectiveness and robustness of the ORBSLAM3 has been improved significantly. With the developed stereo vision-based autonomous navigation system, the lowcost UAV can track the planned trajectory stably to take images of inner surfaces and structures of the pressure vessel. Future work aims to develop a more effective position tracking controller and deploy the proposed approach on a physical low-cost UAV platform. In addition, we plan to develop a UAV swarm to further improve the efficiency of visual inspection of oil and gas pressure vessels.

\section{ACKNOWLEDGMENT}

Thanks are given to the supports from Strathclyde OGTC robotics research team, especially Dr Gordon Dobie, Dr Charles MacLeod, Mr Mark Robertson and Prof Xiutian Yan.

\section{REFERENCES}

[1] L. Yu, E. Yang, P. Ren, C. Luo, G. Dobie, D. Gu, and X. Yan, "Inspection robots in oil and gas industry: a review of current solutions and future trends," in 2019 25th International Conference on Automation and Computing (ICAC). IEEE, 2019, pp. 1-6.

[2] S. Agnisarman, S. Lopes, K. C. Madathil, K. Piratla, and A. Gramopadhye, "A survey of automation-enabled human-in-theloop systems for infrastructure visual inspection," Automation in Construction, vol. 97, pp. 52-76, 2019.

[3] J. Palomba and M. MScEcon, "Unmanned aerial vehicle inspections and environmental benefits," in 15th Asia Pacific Conference for NonDestructive Testing, 2017.

[4] S. Jordan, J. Moore, S. Hovet, J. Box, J. Perry, K. Kirsche, D. Lewis, and Z. T. H. Tse, "State-of-the-art technologies for uav inspections," IET Radar, Sonar \& Navigation, vol. 12, no. 2, pp. 151-164, 2018.

[5] A. R. Castaño, H. Romero, J. Capitán, J. L. Andrade, and A. Ollero, "Development of a semi-autonomous aerial vehicle for sewerage inspection," in Iberian Robotics conference. S Springer, 2019, pp. 7586.

[6] M. Petrlík, T. Báča, D. Heřt, M. Vrba, T. Krajník, and M. Saska, "A robust uav system for operations in a constrained environment," IEEE Robotics and Automation Letters, vol. 5, no. 2, pp. 2169-2176, 2020

[7] T. Özaslan, G. Loianno, J. Keller, C. J. Taylor, V. Kumar, J. M. Wozencraft, and T. Hood, "Autonomous navigation and mapping for inspection of penstocks and tunnels with mavs," IEEE Robotics and Automation Letters, vol. 2, no. 3, pp. 1740-1747, 2017.

[8] S. Kuutti, S. Fallah, K. Katsaros, M. Dianati, F. Mccullough, and A. Mouzakitis, "A survey of the state-of-the-art localization techniques and their potentials for autonomous vehicle applications," IEEE Internet of Things Journal, vol. 5, no. 2, pp. 829-846, 2018.

[9] J. Bian, X. Hui, X. Zhao, and M. Tan, "A point-line-based slam framework for uav close proximity transmission tower inspection," in 2018 IEEE International Conference on Robotics and Biomimetics (ROBIO). IEEE, 2018, pp. 1016-1021.

[10] L. Teixeira, I. Alzugaray, and M. Chli, "Autonomous aerial inspection using visual-inertial robust localization and mapping," in Field and Service Robotics. Springer, 2018, pp. 191-204.

[11] J. Bian, X. Hui, X. Zhao, and M. Tan, "A monocular vision-based perception approach for unmanned aerial vehicle close proximity transmission tower inspection," International Journal of Advanced Robotic Systems, vol. 16, no. 1, p. 1729881418820227, 2019.

[12] J. Nikolic, M. Burri, J. Rehder, S. Leutenegger, C. Huerzeler, and R. Siegwart, "A uav system for inspection of industrial facilities," in 2013 IEEE Aerospace Conference. IEEE, 2013, pp. 1-8.

[13] C. Campos, R. Elvira, J. J. G. Rodríguez, J. M. Montiel, and J. D. Tardós, "Orb-slam3: An accurate open-source library for visual, visualinertial and multi-map slam," arXiv preprint arXiv:2007.11898, 2020

[14] K. Luo, M. Lin, P. Wang, S. Zhou, D. Yin, and H. Zhang, "Improved orb-slam 2 algorithm based on information entropy and image sharpening adjustment," Mathematical Problems in Engineering, vol. 2020, 2020.

[15] K. S. Vepuri and N. Attar, "Improving the performance of deep learning in facial emotion recognition with image sharpening," International Journal of Computer and Information Engineering, vol. 15, no. 4, pp. 234-237, 2021.

[16] F. Gao, X. Xue, J. Sun, J. Wang, and Y. Zhang, "A sar image despeckling method based on two-dimensional s transform shrinkage," IEEE Transactions on Geoscience and Remote Sensing, vol. 54, no. 5, pp. 3025-3034, 2016.

[17] P. Charles et al., "Digital video and hdtv algorithms and interfaces," Morgan Kaufmann Publishers, San Francisco, vol. 260, p. 630, 2003.

[18] S.-C. Huang, F.-C. Cheng, and Y.-S. Chiu, "Efficient contrast enhancement using adaptive gamma correction with weighting distribution," IEEE transactions on image processing, vol. 22, no. 3, pp. 1032-1041, 2012

[19] L. Meier, D. Honegger, and M. Pollefeys, "Px4: A node-based multithreaded open source robotics framework for deeply embedded platforms," in 2015 IEEE international conference on robotics and automation (ICRA). IEEE, 2015, pp. 6235-6240.

[20] M. Quigley, K. Conley, B. Gerkey, J. Faust, T. Foote, J. Leibs, R. Wheeler, and A. Y. Ng, "Ros: an open-source robot operating system," in ICRA workshop on open source software, vol. 3, no. 3.2. Kobe, Japan, 2009, p. 5.

[21] N. Koenig and A. Howard, "Design and use paradigms for gazebo, an open-source multi-robot simulator," in 2004 IEEE/RSJ International Conference on Intelligent Robots and Systems (IROS)(IEEE Cat. No. 04CH37566), vol. 3. IEEE, 2004, pp. 2149-2154. 